\title{
Integrative genomic analysis identified common regulatory networks underlying the correlation between coronary artery disease and plasma lipid levels
}

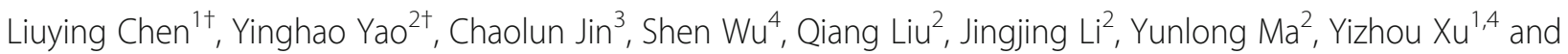
Yigang Zhong ${ }^{4^{*}}$ (D)

\begin{abstract}
Background: Coronary artery disease (CAD) and plasma lipid levels are highly correlated, indicating the presence of common pathways between them. Nevertheless, the molecular pathways underlying the pathogenic comorbidities for both traits remain poorly studied. We sought to identify common pathways and key driver genes by performing a comprehensive integrative analysis based on multi-omic datasets.

Methods: By performing a pathway-based analysis of GWAS summary data, we identified that lipoprotein metabolism process-related pathways were significantly associated with CAD risk. Based on LD score regression analysis of CAD-related SNPS, significant heritability enrichments were observed in the cardiovascular and digestive system, as well as in liver and gastrointestinal tissues, which are the main regulators for lipid level.

Results: We found there existed significant genetic correlation between CAD and other lipid metabolism related traits (the smallest $P$ value $<1 \times 10^{-16}$ ). A total of 13 genes (e.g., LPA, APOC1, APOE and SLC22A3) was found to be overlapped between CAD and plasma lipid levels. By using the data-driven approach that integrated transcriptome information, we discovered co-expression modules associated prominently with both CAD and plasma lipids. With the detailed topology information on gene-gene regulatory relationship, we illustrated that the identified hub genes played important roles in the pathogenesis of CAD and plasma lipid turbulence.

Conclusion: Together, we identified the shared molecular mechanisms underlying the correlation between CAD and plasma lipid levels.
\end{abstract}

Keywords: Coronary artery disease, Lipid levels, Multi-omics, Susceptible genes, Pathways, GWAS

\section{Background}

Coronary artery disease $(\mathrm{CAD})$ is one of the leading causes of death globally [1]. Plasma lipid levels, including lowdensity lipoprotein (LDL) cholesterol, high-density lipoprotein (HDL) cholesterol, triglycerides and total cholesterol, are all associated with regulation of the risk for CAD. Further, The INTERHEART study indicated that $45 \%$ of heart

\footnotetext{
*Correspondence: zhongyigangly@163.com

'Liuying Chen and Yinghao Yao contributed equally to this work.

${ }^{4}$ Department of Cardiology, Affiliated Hangzhou First People's Hospital,

Zhejiang University School of Medicine, Hangzhou, China

Full list of author information is available at the end of the article
}

attacks in Western Europe are due to abnormal blood lipid levels [2].

CAD and blood lipid levels are both heritable, with the genetic contribution estimated to be $40-60 \%$. Genomewide association studies (GWAS) have successfully identified more than hundreds of risk loci for CAD and plasma lipid levels [3-6]. Importantly, several genetic studies also suggest the existence of shared polygenic pleiotropy between CAD and blood lipids $[4,7,8]$. For example, the genes APOA5, TRIB1 and APOC3, which were significantly associated with plasma lipids, also showed prominent risk to $\operatorname{CAD}[9,10]$. However, due to lack of multi-dimensional data integration analysis, the

(c) The Author(s). 2019 Open Access This article is distributed under the terms of the Creative Commons Attribution 4.0 International License (http://creativecommons.org/licenses/by/4.0/), which permits unrestricted use, distribution, and reproduction in any medium, provided you give appropriate credit to the original author(s) and the source, provide a link to the Creative Commons license, and indicate if changes were made. The Creative Commons Public Domain Dedication waiver (http://creativecommons.org/publicdomain/zero/1.0/) applies to the data made available in this article, unless otherwise stated. 
underlying mechanistic insights into the pathogenesis of comorbidity remain largely unknown.

In recent years, large-scale genetic association studies have yielded new insights into the genetic architecture of CAD and blood lipid levels, which enabled us to investigate the genetic etiology of comorbidity. As the fact that pleiotropy is pervasive, many relevant diseases or traits are commonly associated with the same underlying causal variants. The emerging challenge in today research is how to interpret the functional effects of common genetic signals between diseases and their risk factors. In addition, the cell-type-specific regulatory elements that control specific cell functions also increase the difficulties to identify key disease pathways and processes.

In the current investigation, we conducted a systematic analysis with the goal of revealing the underlying genetic architecture of CAD and shared gene regulatory network with plasma lipids using large-scale GWAS summary and gene expression data. By performing a transcriptome analysis in three relevant tissues, we modeled co-expression networks and identified common trait-associated modules shared between CAD and plasma lipids. Finally, we integrated topological gene regulatory networks to identify hub connected genes for both traits.

\section{Methods}

\section{GWAS summary data sets}

\section{Dataset \#1 for coronary artery disease}

We obtained summary statistics from a large GWAS metaanalysis comprising more than 120,000 cases and 339,115 controls (Additional file 2: Table S1) [11]. Complete GWAS summary statistics were downloaded from the CARDIoGRAMplusC4D Consortium website (http://www.cardiogramplusc4d.org/data-downloads/).

\section{Dataset \#2 for plasma lipid levels}

We obtained a published GWAS meta-analysis association data for lipid levels from Center for Statistical Genetics. This study was a joint analysis that examined 188,577 individuals that were genotyped with two platforms from multiple studies [12]. Complete GWAS summary statistics were downloaded from the website (http://csg.sph.umich.edu/willer/public/lipids2013/).

\section{Transcriptome data sets}

There were three gene expression datasets obtained from Gene Expression Omnibus (GEO) database (Additional file 2: Table S2). For GSE30169, we filtered samples treated with $40 \mu \mathrm{g} / \mathrm{ml}$ oxidized 1-palmitoyl-2-arachidonoyl-sn-glycero-3-phosphatidylcholine (Ox-PAPC), which left 307 remaining normal primary human aortic endothelial cells. For GSE7965, adipose tissue samples from 701 individuals were included in the analysis. For GSE24335,
651 samples with expression profile of liver tissue were included in the analysis.

\section{Gene set analysis by GWAS summary statistics}

We used the Multi-marker Analysis of GenoMic Annotation (MAGMA) [13] to test for enrichment of welldocumented gene-sets, including data sources from KEGG, GO, BioCarta [14], and Reactome [15], with CAD. The SNPs were assigned to all protein-coding genes (or within a region extended $-30 \mathrm{~kb}$ upstream and $+10 \mathrm{~kb}$ downstream of the gene) based on the autosome of NCBI 37.3 coordinates. After SNP annotation, there were 18,410 genes containing SNPs in genotype data. For the gene set analysis, we restricted the analysis to 4608 pathways comprising 5-300 genes. MAGMA's built in empirical multiple testing corrections were used to correct raw $P$ values with 10 , 000 permutations.

\section{Partitioning heritability for CAD loci by cell-type-specific annotation}

The polygenic contributions for cell-type-specific functional elements were estimated by linkage disequilibrium (LD) score regression analysis [16]. For CAD summary data, only common SNPs (MAF > 1\%) presented in the HapMap version 3 data set were included in analysis model. LD scores were calculated by the 1000 Genomes Project Phase 1 EUR reference panel. As described by Finucane et al. [16], we first created a "full baseline model" with a total number of 53 overlapping functional categories. For cell type specific analysis, we used annotations from ten groups, including adrenal/pancreas, central nervous system (CNS), cardiovascular, connective/bone, gastrointestinal, immune/hematopoietic, kidney, liver, skeletal muscle, and others.

\section{Tissue/cell-type expression enrichment analysis}

DEPICT analysis (Data-Driven Expression-Prioritized Integration for Complex Traits) [17] was used to test for enrichment of tissues or cell types where CAD-related gene are highly expressed. Firstly, we used PLINK v1.07 [18] to identify independent SNPs with $P$ value less than $1 \times 10^{-5}$ from CAD GWAS summary, LD information was provided by the 1000 Genomes Project Phase 1 EUR reference panel. Then, we took advantage of the build-in data sets from DEPICT consisting of 209 tissue/cell types assembled from 37,427 human microarray samples for expression enrichment analysis.

\section{Genetic correlation analysis}

We used the LD score regression method $[19,20]$ to profile the pattern of genetic correlations between CAD and lipid metabolism related traits, including low-density lipoprotein (LDL) cholesterol, high-density lipoprotein (HDL) cholesterol, triglycerides, total cholesterol, BMI, and waist-hip ratio. Quality control steps were adopted from LD scores 
default procedures, including imputation quality $>0.9$ and MAF $>0.1$. Moreover, all SNPs retained to analysis were merged to SNPs in HapMap 3 reference panel.

\section{Building gene co-expression network modules}

CAD and lipid metabolism related tissues (including liver, aortic endothelial cells and adipose) transcriptome data were obtained from GEO datasets (https://www.ncbi.nlm. nih.gov/gds/) (Additional file 2: Table S2). Low-expressed and non-varying genes in each dataset were filtered to avoid noise, results in an average of 12,000 genes to be included in the following analysis. We applied the Weighted Correlation Network Analysis (WGCNA) [21] to construct gene co-expression modules. A number of 30 were set for minimum module size. We chose 0.1 as cut line in the dendrogram to merge similar modules (corresponding to correlation of 0.9 ).

\section{Identification of co-expression modules with over- representation of genetic association signals}

We conducted Marker Set Enrichment Analysis (MSEA) to identify genetically perturbed co-expression modules for each phenotype using the Mergeomics pipeline [22, 23]. For the current analysis, MSEA takes three elements into workflow: (1) summary data for each GWAS (CAD, HDL, LDL, TC, TG, BMI, and WHRadjBMI); (2) assignment of SNPs to their corresponding genes; (3) functionally related gene sets generated from co-expression module.

\section{Identification of hub genes using weighted key driver analysis (wKDA)}

The Mergeomics pipeline offers a function to detect key drivers and hub genes using detailed topology information on gene regulatory relationships [23]. We used GIANT networks [24] from three tissues (aorta, adipose and liver), which provide detailed interactions between genes according to independent gene expression datasets and protein interaction information. All genes in the CAD-associated module that also showed nominal significance in lipid metabolism related traits $(P<0.1)$ were mapped into GIANT networks with edges information, which support tissue-specific function interactions.

\section{Results}

CAD associated pathways are enriched in lipoprotein metabolism processes

To reveal the genetic architecture of CAD, we first performed pathway analysis to test the associations of predefined functional gene-sets, including KEGG, GO, BioCarta, and Reactome (see Methods for details). 4608 pathways with a size 5-300 genes per pathway were retained for downstream analyses in consideration of appropriate specificity and high efficiency. After corrections for multiple testing by permutation tests, 12 significant enriched pathways with corrected $P$ value $<0.05$ were identified (Additional file 2: Table S3). The top ranked pathway was collagen type IV $\left(P=1.32 \times 10^{-09}\right)$, consisting of 6 genes coding type IV collagen proteins. Notably, two-thirds of pathways reached significant associations involved in lipoprotein metabolism and cholesterol and triglyceride homeostasis. These pathways contained 10 common genes, i.e., LDLR, LPA, PLG, APOE, LIPA, LPL, APOB, ABCG8, $A B C G 5, A P O C 4$ (Additional file 2: Table S4), that were significantly associated with $\mathrm{CAD}\left(P<2.72 \times 10^{-06}\right)$ by using the MAGMA analysis.

\section{CAD related SNPs/genes were functionally annotated at liver and gastrointestinal tissues}

We applied stratified LD score regression to estimate the global enrichment of heritability contributed by CAD related risk SNPs in 53 genomics features annotated from 10 cell type groups. Large and significant enrichments were observed for the cardiovascular and digestive systems. For cardiovascular tissues, 11.1\% SNPs explained an estimated $52.0 \%$ SNP-heritability $\left(P=1.12 \times 10^{-08}\right.$ for enrichment analysis). Liver and gastrointestinal tissues showed 4.63 and 3.49 fold enrichment $\left(P<1 \times 10^{-06}\right.$; Fig. 1), respectively. The significant heritability enrichment contributed by liver and gastrointestinal tissues was in line with our GWAS-based pathway analysis, highlighting that lipoprotein metabolism and cholesterol and triglyceride homeostasis contribute a genetic risk to CAD. Furthermore, DEPICT framework identified multiple tissues in the digestive system where genes from CAD-associated loci were highly expressed (Fig. 2; Additional file 2: Table S5). Although the significance level failed to pass multiple tests correction, we observed a significant enrichment of digestive system among all tissues/cell-type (14/42, Fisher's exact $P=0.03$ ).

\section{Genetic correlations between CAD and lipid metabolism related traits}

Our pathway and functional enrichment analysis emphasized the crucial role of liver and gastrointestinal tissues in the pathology of CAD. These tissues are the main ones for producing various lipids in blood, which include low-density lipoprotein (LDL) cholesterol, high-density lipoprotein (HDL) cholesterol, triglycerides, and total cholesterol; all of them have been demonstrated to be risk factors for CAD. Thus, the shared genetic and molecular regulatory mechanisms between CAD and lipid metabolism related traits were warranted to be studied.

We also downloaded GWAS summary data for four blood lipid level measurements from the Center for Statistical Genetics (see Methods). Moreover, BMI and waist-hip ratio GWAS data from Genetic Investigation of ANthropometric Traits (GIANT) were also included. Genetic correlations were calculated between CAD and 


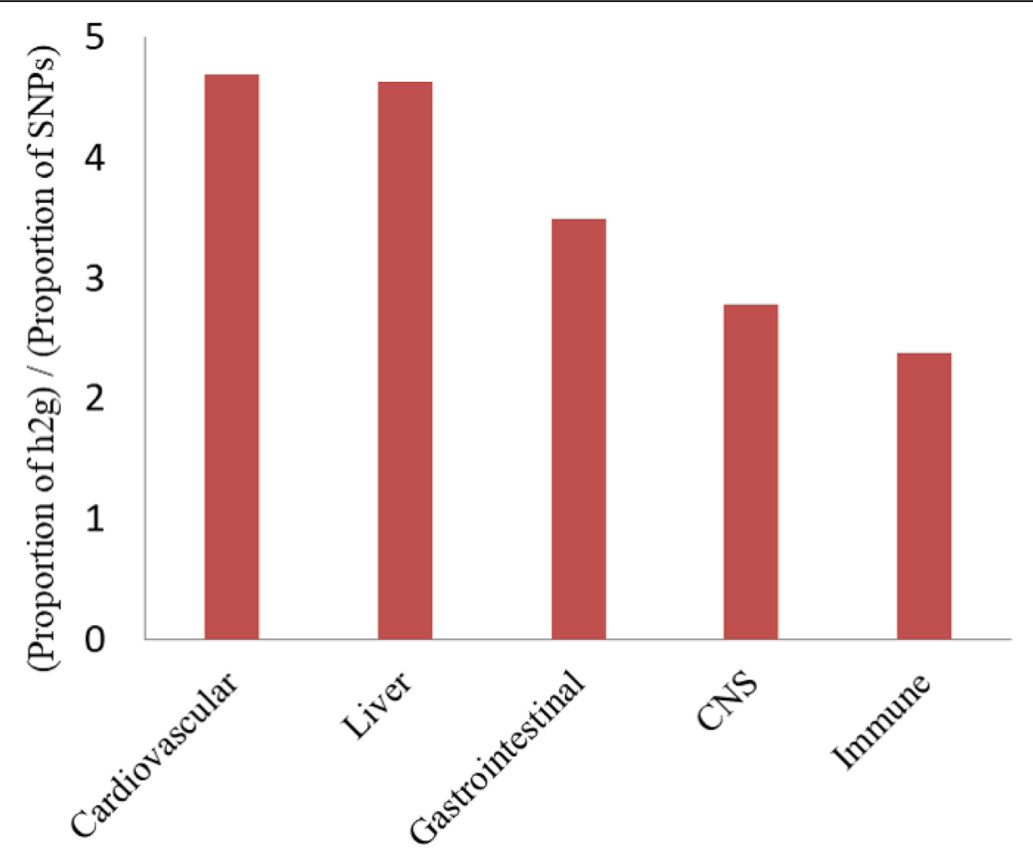

Fig. 1 Heritability enrichment of cell type groups and SNPs and genes related to CAD functioned in cardiovascular and digestive tissues. Vertical axis represents enrichment fold that calculated by proportion of heritability divided by proportion of SNPS

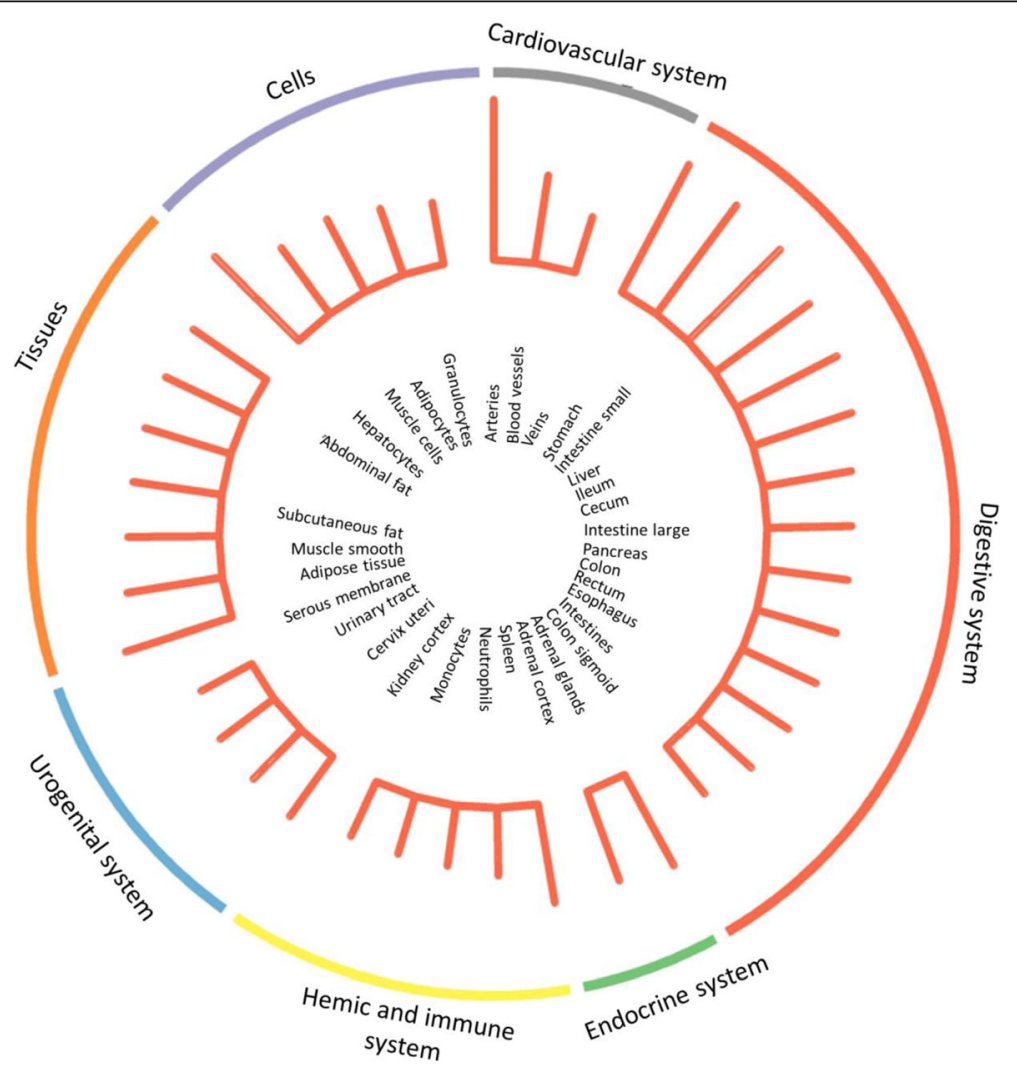

Fig. 2 DEPICT identifies Cardiovascular and Digestive system where genes from CAD-associated loci are highly expressed. Each sector represents an organ tissue, bar length indicates the Log2(P-value) for that cell type or tissue 
all six traits using LD Score regression. Significant genetic correlations were found between CAD and all the other investigated traits with smallest $P$ value less than $1 \times 10^{-16}$. We found that CAD was negatively associated with HDL-C $(r=-0.30)$, but showed positive correlations with LDL-C, TC, TG, BMI and WHRadjBMI (Fig. 3).

\section{CAD and lipid metabolism related traits shared common} genetic association signals

To further reveal the underlying biological mechanisms of the comorbidity between CAD and plasma lipid levels, we made a direct comparison using gene-based association signals. Gene-based association signals of genes that showed significant associations with $\mathrm{CAD}\left(P<2.72 \times 10^{-6}\right)$ were compared to that of nominally significant genes related to plasma lipid levels $(P<0.05$, Fig. 4$)$. We discovered that 13 genes overlapped across all five phenotypes (Gene set \#1 in Additional file 2: Table S6). Notably, the common signals, including $A P O C 1, A P O E$, and $A P O B$, of the apolipoprotein family, which were highly expressed in the liver and played crucial roles in lipoprotein metabolism. The PLG gene encodes a secreted blood zymogen that is primarily expressed in liver tissue, and abnormality of this gene contributes susceptibility to thrombophilia [25].

In addition, we compared significantly CAD-enriched pathways (FDR $<0.05)$ with enriched pathways related to plasma lipid levels. Among these 12 top enriched pathways for CAD, 11 also showed significances among plasma lipid level traits $(\mathrm{P}<0.05)$. Of them, three enriched pathways, including cholesterol homeostasis, chylomicron mediated lipid transport, and lipoprotein metabolism, maintained statistical significance after multiple testing corrections across all five traits (Table 1). Importantly, there were 6 genes $(6 / 13)$ that belong to the shared Gene set \#1 that also appeared in these common pathways.

Furthermore, we sought to determine whether the 13 shared genes from gene-based analysis were significantly overrepresented in these common pathways. A random resampling of the same number of genes for the shared genes was conducted 10 million times. All genes within our predefined pathways $(N=16,994)$ served as the pool for our randomization test. After the randomization trials, we observed no instances of any overlap greater than the real one that contains 6 overlaps (Additional file 2: Table S7).

\section{Identification of co-expression modules genetically associated with CAD and blood lipid levels}

We investigated the expression profiles of co-expression modules that associated with genetic markers in various tissues relevant to CAD and lipid metabolism by Marker Set Enrichment Analysis (MSEA). Briefly, co-expression networks were constructed using transcriptome datasets from liver, aortic, endothelial cells and adipose, respectively (Additional file 1: Figures S1, S2, and S3). The identified modules were used as functionally related gene sets to enter into MSEA and the significance of enrichment of a co-expression module to potential functional disease SNPs defined by GWAS was assessed using Chi-square-like statistics. For each tissue or cell type, we prioritized common modules that not only significantly associated with $\mathrm{CAD}(\mathrm{FDR}<0.05)$, but also

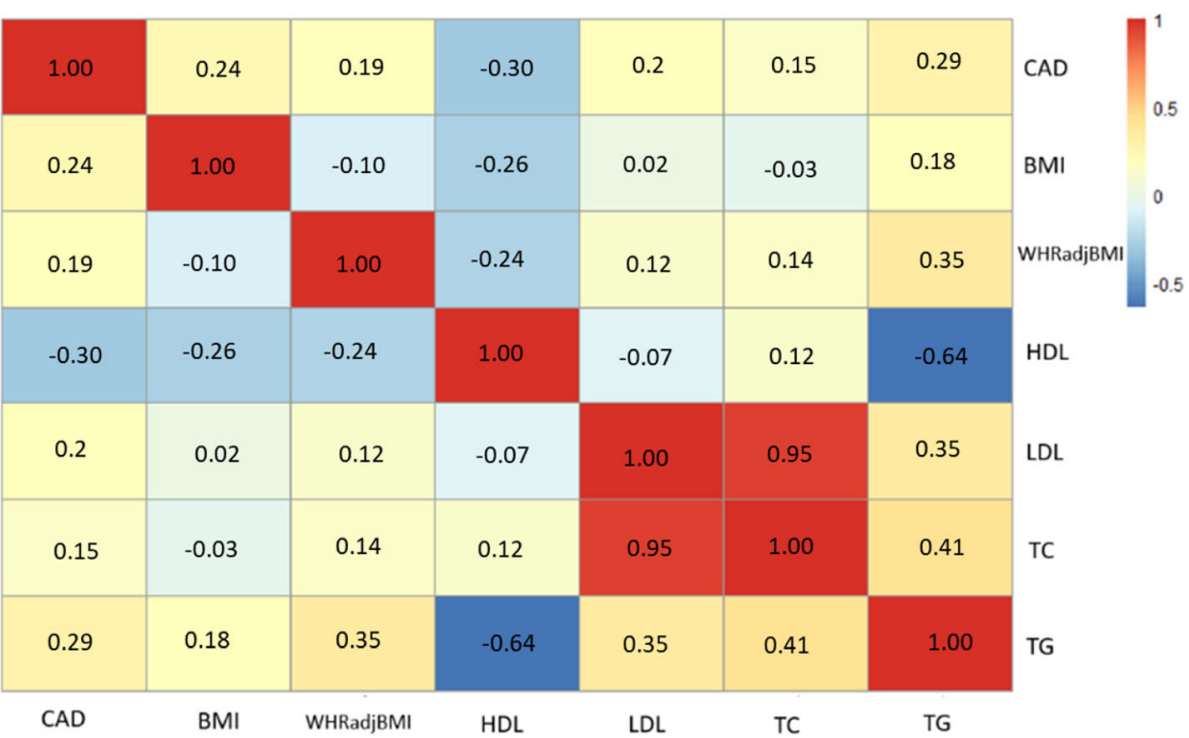

Fig. 3 Genetic correlation between CAD and six other phenotypes (BMI, WHRadjBMI, HDL, LDL, TC, and TG). Red color represents for positive correlations and blue color represents negative correlations 


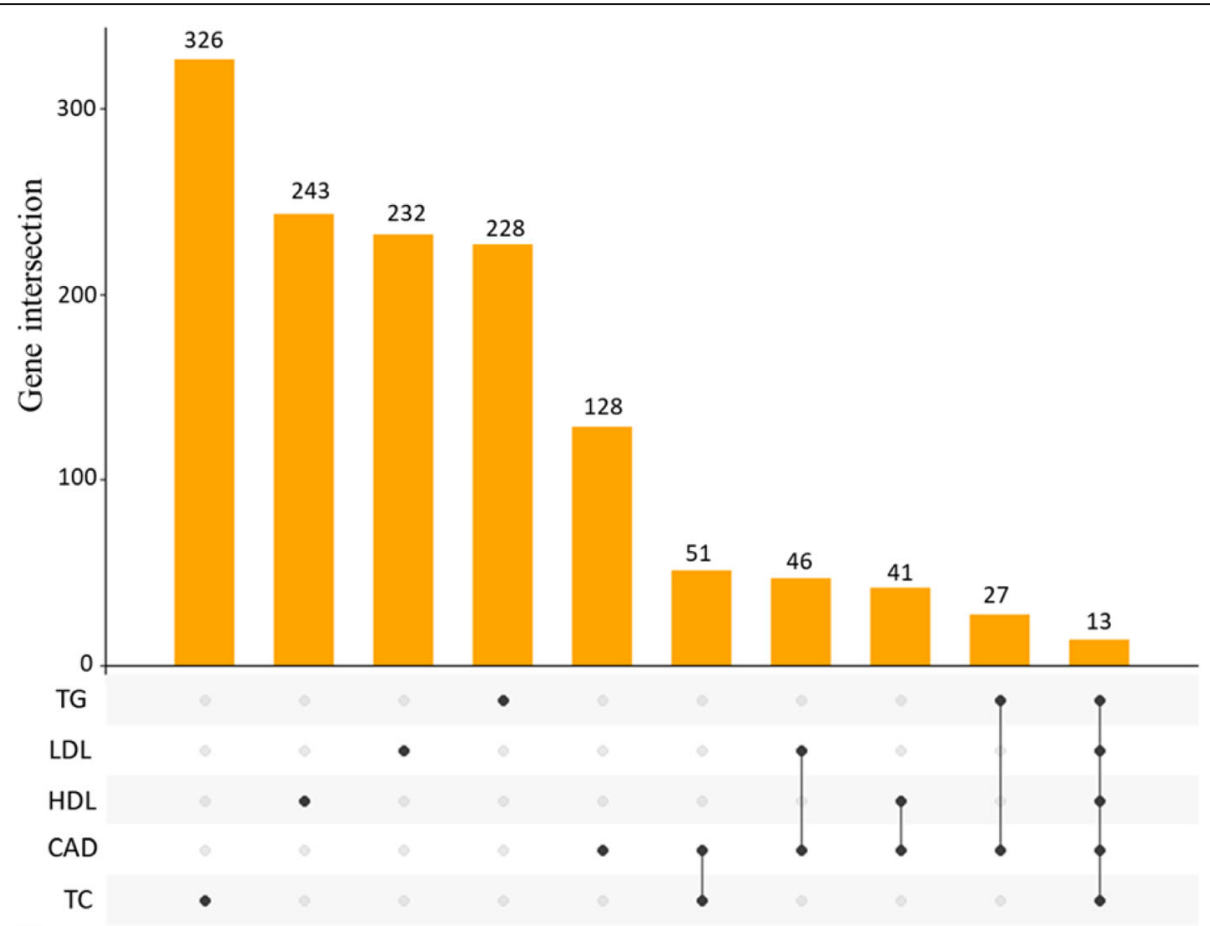

Fig. 4 UpSetR plot shows the overlap of significantly genes discovered from MAGMA gene-based analysis between CAD and four lipid metabolism related traits

associated with at least one blood lipid traits (Fig. 5). In Aortic endothelial cells (Fig. 5a), two modules were significantly associated with CAD $\left(\mathrm{P}_{\text {Turquoise }}=3.51 \times 10^{-4}\right.$, $\left.P_{\text {Yellow }}=0.013\right)$. Turquoise additionally associated with HDL $\left(P=2.35 \times 10^{-6}\right)$, LDL $\left(P=3.74 \times 10^{-4}\right)$, TC $(P=$ $\left.6.04 \times 10^{-5}\right)$ and TG $\left(P=2.12 \times 10^{-3}\right)$. In adipose tissue, a light yellow module was associated with both CAD and BMI (Fig. 5b), and a blue module was associated both CAD and HDL (Fig. 5b). One module reached significance in liver tissue (Fig. 5c).

\section{Prioritization of hub genes for common modules}

The common co-expression module identified above mainly provided expression patterns without detailed topology information on gene-gene regulatory relationship. By applying a wKDA analysis that integrates GIANT network, we prioritized hub genes within the common modules in three relevant tissues. Together, our analysis identified 571, 2843, and 3016 significantly changed genes with FDR $<0.01$ in adipose, aortic endothelial cells and liver tissue, respectively. Of them, we

Table 1 Common pathways between CAD and plasma lipid levels

\begin{tabular}{llllll}
\hline Pathway name & P-CAD & P-HDL & P-LDL & P-TC & P-TG \\
\hline Positive regulation of cholesterol storage & $3.99 \times 10^{-09}$ & $8.07 \times 10^{-22}$ & $9.38 \times 10^{-03}$ & $4.45 \times 10^{-03}$ & $2.03 \times 10^{-12}$ \\
Apolipoprotein binding & $1.10 \times 10^{-06}$ & $8.25 \times 10^{-29}$ & $1.82 \times 10^{-05}$ & $1.99 \times 10^{-18}$ & $5.35 \times 10^{-09}$ \\
low-density lipoprotein particle & $8.32 \times 10^{-09}$ & $2.13 \times 10^{-05}$ & $1.23 \times 10^{-10}$ & $8.16 \times 10^{-06}$ & $2.10 \times 10^{-03}$ \\
Lipoprotein catabolic process & $2.72 \times 10^{-09}$ & $9.06 \times 10^{-12}$ & $3.30 \times 10^{-12}$ & $6.44 \times 10^{-11}$ & $1.12 \times 10^{-07}$ \\
Chylomicron & $2.31 \times 10^{-06}$ & $3.32 \times 10^{-11}$ & $6.99 \times 10^{-10}$ & $5.61 \times 10^{-07}$ & $9.59 \times 10^{-21}$ \\
Cholesterol homeostasis* & $9.61 \times 10^{-08}$ & $4.83 \times 10^{-17}$ & $6.99 \times 10^{-15}$ & $3.49 \times 10^{-26}$ & $4.17 \times 10^{-12}$ \\
Reverse cholesterol transport & $4.13 \times 10^{-06}$ & $3.76 \times 10^{-23}$ & $8.02 \times 10^{-05}$ & $1.25 \times 10^{-20}$ & $2.54 \times 10^{-07}$ \\
Triglyceride homeostasis & $3.65 \times 10^{-07}$ & $3.32 \times 10^{-18}$ & $6.03 \times 10^{-06}$ & $5.40 \times 10^{-10}$ & $2.23 \times 10^{-20}$ \\
Lipase & $6.16 \times 10^{-07}$ & $2.82 \times 10^{-12}$ & $2.99 \times 10^{-02}$ & $1.22 \times 10^{-05}$ & $1.77 \times 10^{-08}$ \\
Chylomicron mediated lipid transport* & $4.81 \times 10^{-06}$ & $6.34 \times 10^{-14}$ & $5.37 \times 10^{-12}$ & $1.72 \times 10^{-17}$ & $1.16 \times 10^{-18}$ \\
Lipoprotein metabolism * & $7.59 \times 10^{-08}$ & $1.10 \times 10^{-17}$ & $6.29 \times 10^{-11}$ & $8.01 \times 10^{-19}$ & $2.25 \times 10^{-18}$ \\
\hline
\end{tabular}

The asterisk (*) marked pathways maintained significance after multiple testing corrections across all five traits 


\begin{tabular}{|c|c|c|c|c|c|c|c|}
\hline \multirow[b]{3}{*}{$\mathrm{A}$} & & & & & & & \multirow{2}{*}{$\begin{array}{l}\text { FDR }<0.05 \\
\text { Raw } \mathrm{P}<0.1\end{array}$} \\
\hline & & & & & & & \\
\hline & 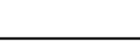 & & & & & & \\
\hline \multicolumn{8}{|c|}{ Aortic endothelial cells } \\
\hline Module ID & CAD_P & HDL_P & LDL_P & TC_P & TG_P & BMI_P & WHRadjBMI_P \\
\hline Turquoise & $3.51 \mathrm{E}-04$ & $2.35 \mathrm{E}-06$ & $3.74 \mathrm{E}-04$ & $6.04 \mathrm{E}-05$ & $2.12 \mathrm{E}-03$ & 0.024 & $8.54 \mathrm{E}-03$ \\
\hline Yellow & 0.013 & $3.79 \mathrm{E}-04$ & 0.114 & NA & 0.092 & 0.027 & 0.067 \\
\hline \multirow{2}{*}{\multicolumn{8}{|c|}{$\mathrm{B}$ Adipose tissue }} \\
\hline & & & & & & & \\
\hline Module ID & CAD_P & HDL_P & LDL_P & TC_P & TG_P & BMI_P & WHRadjBMI_P \\
\hline Lightyellow & $2.45 \mathrm{E}-05$ & 0.455 & 0.996 & 0.825 & 0.012 & $3.65 \mathrm{E}-03$ & NA \\
\hline Blue & $1.04 \mathrm{E}-03$ & 0.010 & 0.327 & 0.458 & 0.058 & 0.465 & 0.130 \\
\hline \multirow{2}{*}{\multicolumn{8}{|c|}{$\mathrm{C}$ Liver tissue }} \\
\hline & & & & & & & \\
\hline Module ID & CAD_P & HDL_P & LDL_P & TC_P & TG_P & BMI_P & WHRadjBMI_P \\
\hline Turquoise & $1.55 \mathrm{E}-03$ & 0.027 & 0.172 & 0.187 & $4.92 \mathrm{E}-03$ & 0.173 & 0.102 \\
\hline
\end{tabular}

Fig. 5 CAD associated modules show significances among lipid metabolism related traits in relevant tissues. a for aortic endothelial cell; $\mathbf{b}$ for adipose tissue; $\mathbf{c}$ for liver tissue. Red box corresponds FDR $<0.05$ and pink box corresponds $P$ value $<0.1$

revealed 245 key drivers (KDs) that showed significance in all three tissues for both CAD and plasma lipid associated modules (Additional file 2: Table S8).

\section{Discussion}

The present study systematically investigated the molecular links between CAD and plasma lipid levels by integration of GWAS signals with gene expression data. Our results showed that the genetic contribution of CAD is heavily concentrated in cell-type-specific regulatory regions of the cardiovascular and digestive systems, the sites of regulating plasma lipid level. Common genes and pathways were used to detect the effects of pleiotropy within the comorbidity between the two traits of interests. Data driven analysis of transcriptome sequences in three relevant tissues modeled co-expression networks that were significantly associated with both CAD and plasma lipids. A gene regulatory network helped to prioritized hub genes that were strongly connected in sub-networks.

Previous studies [26-28] have documented multiple lines of evidences to support the comorbidity between dyslipidemia and cardiovascular disease. The seminal finding of Framingham Heart Study showed that plasma cholesterol concentration was associated with potential CAD risk. The following randomized controlled trail (RCT) also demonstrates the causal relationship between CAD and plasma lipid levels. Evidence from human genetic studies identified loss-of-function mutations in the $L D L R$ genes to be associated with high level of plasma LDL-C and premature CAD. Genes that modulate plasma triglyceride levels have been associated with CAD risk. Combined, these genetic findings suggest that lipoprotein and triglyceride-rich lipoproteins contribute to CAD. All these abovementioned gene have also been detected in our analysis, which provides an independent support to those reported findings.

In the current study, our findings provided further evidence of the underlying genetic link between CAD and plasma lipoproteins. The top enriched pathways detected in this study included lipoprotein catabolism process, positive regulation of cholesterol storage, lipoprotein metabolism, and cholesterol and triglyceride homeostasis. Notably, 8 out of 11 pathways that passed significance after corrections for multiple tests were found to be involved in the regulation of plasma lipids levels. Besides the cardiovascular system, we detected enrichments in liver and gastrointestinal tissues. In addition, DEPICT also indicated digestive system, including upper gastrointestinal tract, stomach, intestine small, hepatocytes, liver, ileum, and cecum, as the most relevant tissues where CAD risk genes 
highly expressed. These findings were consistent with a recent GWAS study using UK biobank samples [29].

Moreover, we found significant genetic correlations between CAD and plasma lipids based on LD score regression analysis. Our results showed that CAD was negatively correlated with HDL-C and positively correlated with LDL-C. HDL-C particles remove fats and cholesterol from cells; individuals with higher levels of HDL-C are less likely to suffer from cardiovascular diseases [30]. Inversely, LDL-C particles used as a risk factor for CAD; individuals with lower levels of LDL-C are more likely to reduce the risk of major coronary events and coronary death [31-33]. A recent epidemiology study using 4205 new-onset patients with stable CAD in Chinese population discovered that plasma HDL-C levels appear to be a predicator of coronary severity [34]. LDL-C and triglyceride-rich lipoproteins were previously treated as casual biomarkers for CAD [35].

One of the main findings in the current study pinpointed common genes and pathways implicated in the comorbidity between $C A D$ and plasma lipids level. Our gene-based analysis found that $L D L R, A P O B$, and PCSK9 were significantly associated with both CAD and LDL-C. These three genes participate in the cellular LDL particle uptake, promote degradation of LDL particles, and reduce the risk of CAD [36]. The detected shared pathways unveiled by our systematic analysis included lipoprotein metabolism processes, which also provide evidence of the shared genetic vulnerability between the two traits of interests.

The systematically integrative pipeline by using multiomics data could help us to better understand the biological mechanisms of complex diseases or comorbidities. The computational pipeline of Mergeomics combines disease-related genetic association data with pre-defined sets of connected genes to identify key drivers that are enriched for genes in the cellular regulatory network [13]. By identifying genetically-driven CAD and plasma lipids modules independently, we found that CAD related modules also exhibited significance in plasma lipids. Importantly, the CAD associated gene sets coincidently linked with plasma lipids in a tissue specific manner. The combination of univariate association signals with expression data in relevant tissues made the shared regulatory network more explicit.

By applying a comprehensive network modeling system, we identified several critical key modulators that have highly pathogenic potential for CAD. These key-drivers (KDs) were enriched in both CAD and plasma lipids associated co-expression modules, which were tissue-specific regulated. Further, we presented the sub-networks where KDs regulated many known disease genes for both CAD and plasma lipids. The gene-gene interactions or networks modules, which constructed from other independent studies, implied more comprehensive conditions that to unveil biological insights [37, 38]. We identified common KDs in three CAD-relevant tissues, indicating the crucial role of these genes implicated in the comorbidity of between $\mathrm{CAD}$ and plasma lipid levels.

There are several limitations in the current study. First, the data-driven analysis was constrained by the current available functional datasets. For the missing information, we expected further investigation from additional relevant tissues with multidimensional functional annotation data. Second, the inferred gene-gene interactions derived from KDs in our subnetworks need further experimental validation at various levels. The regulatory effects of KDs on neighboring genes warrant future investigation using independent in vivo and in vitro systems.

\section{Conclusions}

In sum, the current study revealed the genetic landscape of CAD with functional enrichment of risk loci in lipoprotein metabolism processes and relevant tissues and cell types. Through integrative genetic and expression data, we identified the shared pathogenesis of CAD and plasma lipid traits, including common genes, pathways, and key molecular drivers. This systematic approach provides novel insight into basic pathogenic mechanism for cardiometabolic diseases and relevant comorbidities.

\section{Supplementary information}

Supplementary information accompanies this paper at https://doi.org/10. 1186/s12872-019-01271-9.

Additional file 1: Figure S1. Clustering dendrogram of genes for GSE30169, together with assigned module colors, Figure S2. Clustering dendrogram of genes for GSE7965, together with assigned module colors, Figure S3. Clustering dendrogram of genes for GSE24335, together with assigned module colors. (PPTX 79 kb)

Additional file 2: Table S1. GWAS summary association statistics used in current analysis. Table S2 Transcriptome data used in current analysis. Table S3. Top pathways identified by summarized CAD GWAS data. Table S4. Ten genes that reached genome-wide significance in lipoprotein metabolism, cholesterol and triglyceride homeostasis pathways. Table S5. Tissues that showed nominal significance $(P<0.05)$ revealed by DEPICT tissue/cell type enrichment analysis. Table $\mathbf{S 6 .} 13$ common genes between CAD and plasma lipid related traits. Table S7. Randomization results for 13 shared genes in 3 common pathways that comprising with 85 genes. Table S8. The identification of 245 key driver genes for both CAD and plasma lipid associated modules.

\footnotetext{
Abbreviations

CAD: Coronary artery disease; CNS: Central nervous system; DEPICT: Datadriven expression-prioritized integration for complex traits; GEO: Gene expression omnibus; GIANT: Genetic investigation of Anthropometric traits; GWAS: Genome-wide association study; HDL: High-density lipoprotein; KEGG: Kyoto encyclopedia of genes and genomes; LD: Linkage disequilibrium; LDL: Low-density lipoprotein; MAGMA: The multi-marker analysis of GenoMic annotation; MSEA: Marker set enrichment analysis; SNP: Single nucleotide polymorphism; WGCNA: The weighted correlation network analysis; wKDA: Weighted key driver analysis
} 


\section{Authors' contributions}

$L C$ and YY conducted data collection and bioinformatics analysis and wrote the manuscript. CJ, SW, QL, and JL involved in data collection and reviewed the manuscript. $Y M, Y X$, and $Y Z$ conceived the study and helped to write the manuscript. All authors approved the final draft of the paper.

\section{Funding}

The authors acknowledge the support of two grants from the Natural Science Foundation of Zhejiang Province (LY15H070001) and the China Postdoctoral Science Foundation (2018 M630667). The funders had no role in the designing and conducting of this study and collection, analysis, and interpretation of data and in writing the manuscript.

\section{Availability of data and materials}

Data used in the current investigation are available from public database. CAD GWAS summary data are downloaded from http://www. cardiogramplusc4d.org/data-downloads/. Plasma lipid GWAS summary data are downloaded from http://csg.sph.umich.edu/willer/public/lipids2013/. Gene expression datasets (Accession Nos. GSE30169, GSE7965, and GSE24335) are available from GEO website: https://www.ncbi.nlm.nih.gov/ geo/.

\section{Ethics approval and consent to participate}

Not applicable.

\section{Consent for publication}

Not applicable.

\section{Competing interests}

The authors declare that they have no competing interests.

\section{Author details}

'Zhejiang Chinese Medical University, Hangzhou, China. ${ }^{2}$ State Key Laboratory for Diagnosis and Treatment of Infectious Diseases, The First Affiliated Hospital, Collaborative Innovation Center for Diagnosis and Treatment of Infectious Diseases, Zhejiang University School of Medicine, Hangzhou, China. ${ }^{3}$ Nanjing Medical University, Nanjing, China. ${ }^{4}$ Department of Cardiology, Affiliated Hangzhou First People's Hospital, Zhejiang University School of Medicine, Hangzhou, China.

\section{Received: 1 October 2019 Accepted: 21 November 2019}

\section{Published online: 23 December 2019}

\section{References}

1. Mortality GBD, Causes of Death C. Global, regional, and national life expectancy, all-cause mortality, and cause-specific mortality for 249 causes of death, 1980-2015: a systematic analysis for the global burden of disease study 2015. Lancet. 2016;388(10053):1459-544.

2. Yusuf S, Hawken S, Ounpuu S, Dans T, Avezum A, Lanas F, McQueen M, Budaj A, Pais P, Varigos J, et al. Effect of potentially modifiable risk factors associated with myocardial infarction in 52 countries (the INTERHEART study): case-control study. Lancet. 2004;364(9438):937-52.

3. Erdmann J, Kessler T, Munoz Venegas L, Schunkert H. A decade of genomewide association studies for coronary artery disease: the challenges ahead. Cardiovasc Res. 2018;114(9):1241-57.

4. Teslovich TM, Musunuru K, Smith AV, Edmondson AC, Stylianou IM, Koseki M, Pirruccello JP, Ripatti S, Chasman DI, Willer CJ, et al. Biological, clinical and population relevance of 95 loci for blood lipids. Nature. 2010;466(7307): 707-13.

5. Asselbergs FW, Guo Y, van Iperen EP, Sivapalaratnam S, Tragante V, Lanktree MB, Lange LA, Almoguera B, Appelman YE, Barnard J, et al. Large-scale gene-centric meta-analysis across 32 studies identifies multiple lipid loci. Am J Hum Genet. 2012;91(5):823-38.

6. Liu DJ, Peloso GM, Yu H, Butterworth AS, Wang X, Mahajan A, Saleheen D, Emdin C, Alam D, Alves AC, et al. Exome-wide association study of plasma lipids in >300,000 individuals. Nat Genet. 2017;49(12):1758-66

7. Do R, Willer CJ, Schmidt EM, Sengupta S, Gao C, Peloso GM, Gustafsson S, Kanoni S, Ganna A, Chen J, et al. Common variants associated with plasma triglycerides and risk for coronary artery disease. Nat Genet. 2013:45(11): $1345-52$.
8. LeBlanc M, Zuber V, Andreassen BK, Witoelar A, Zeng L, Bettella F, Wang Y, McEvoy LK, Thompson WK, Schork AJ, et al. Identifying novel gene variants in coronary artery disease and shared genes with several cardiovascular risk factors. Circ Res. 2016;118(1):83-94.

9. Voight BF, Peloso GM, Orho-Melander M, Frikke-Schmidt R, Barbalic M, Jensen MK, Hindy G, Holm H, Ding EL, Johnson T, et al. Plasma HDL cholesterol and risk of myocardial infarction: a mendelian randomisation study. Lancet. 2012;380(9841):572-80.

10. Consortium CAD, Deloukas $P$, Kanoni S, Willenborg C, Farrall M, Assimes $T L$, Thompson JR, Ingelsson E, Saleheen D, Erdmann J, et al. Large-scale association analysis identifies new risk loci for coronary artery disease. Nat Genet. 2013;45(1):25-33

11. Nelson CP, Goel A, Butterworth AS, Kanoni S, Webb TR, Marouli E, Zeng L, Ntalla I, Lai FY, Hopewell JC, et al. Association analyses based on false discovery rate implicate new loci for coronary artery disease. Nat Genet. 2017;49(9):1385-91.

12. Willer CJ, Schmidt EM, Sengupta S, Peloso GM, Gustafsson S, Kanoni S, Ganna A, Chen J, Buchkovich ML, Mora S, et al. Discovery and refinement of loci associated with lipid levels. Nat Genet. 2013:45(11):1274-83.

13. de Leeuw CA, Mooij JM, Heskes T, Posthuma D. MAGMA: generalized geneset analysis of GWAS data. PLoS Comput Biol. 2015;11(4):e1004219.

14. Nishimura D. BioCarta. Biotech Softw Internet Rep Comput Softw J Sci. 2001;2(3):117-20

15. Joshi-Tope G, Gillespie M, Vastrik I, D'Eustachio P, Schmidt E, de Bono B, Jassal B, Gopinath G, Wu G, Matthews L. Reactome: a knowledgebase of biological pathways. Nucleic Acids Res. 2005;33(suppl_1):D428-32.

16. Gusev A, Lee SH, Trynka G, Finucane H, Vilhjalmsson BJ, Xu H, Zang C, Ripke S, Bulik-Sullivan B, Stahl E, et al. Partitioning heritability of regulatory and cell-type-specific variants across 11 common diseases. Am J Hum Genet. 2014:95(5):535-52

17. Pers TH, Karjalainen JM, Chan Y, Westra HJ, Wood AR, Yang J, Lui JC, Vedantam S, Gustafsson S, Esko T, et al. Biological interpretation of genomewide association studies using predicted gene functions. Nat Commun. 2015;6:5890.

18. Purcell S, Neale B, Todd-Brown K, Thomas L, Ferreira MA, Bender D, Maller J, Sklar P, de Bakker PI, Daly MJ, et al. PLINK: a tool set for whole-genome association and population-based linkage analyses. Am J Hum Genet. 2007; 81(3):559-75.

19. Bulik-Sullivan BK, Loh PR, Finucane HK, Ripke S, Yang J, Schizophrenia Working Group of the Psychiatric Genomics C, Patterson N, Daly MJ, Price AL, Neale BM. LD Score regression distinguishes confounding from polygenicity in genomewide association studies. Nat Genet. 2015;47(3):291-5.

20. Bulik-Sullivan B, Finucane HK, Anttila V, Gusev A, Day FR, Loh PR, ReproGen C, Psychiatric Genomics C, Genetic Consortium for Anorexia Nervosa of the Wellcome Trust Case Control C, Duncan L, et al. An atlas of genetic correlations across human diseases and traits. Nat Genet. 2015;47(11):1236-41.

21. Langfelder P, Horvath S. WGCNA: an R package for weighted correlation network analysis. BMC Bioinformatics. 2008;9:559.

22. Shu L, Zhao Y, Kurt Z, Byars SG, Tukiainen T, Kettunen J, Orozco LD, Pellegrini M, Lusis AJ, Ripatti S, et al. Mergeomics: multidimensional data integration to identify pathogenic perturbations to biological systems. BMC Genomics. 2016;17(1):874.

23. Shu L, Chan KHK, Zhang G, Huan T, Kurt Z, Zhao Y, Codoni V, Tregouet DA, Cardiogenics C, Yang J, et al. Shared genetic regulatory networks for cardiovascular disease and type 2 diabetes in multiple populations of diverse ethnicities in the United States. PLoS Genet. 2017;13(9):e1007040.

24. Greene CS, Krishnan A, Wong AK, Ricciotti E, Zelaya RA, Himmelstein DS, Zhang R, Hartmann BM, Zaslavsky E, Sealfon SC, et al. Understanding multicellular function and disease with human tissue-specific networks. Nat Genet. 2015:47(6):569-76.

25. Hellgren M, Egberg N, Eklund J. Blood coagulation and fibrinolytic factors and their inhibitors in critically ill patients. Intensive Care Med. 1984;10(1):23-8.

26. Emerging Risk Factors C, Di Angelantonio E, Sarwar N, Perry P, Kaptoge S, Ray KK, Thompson A, Wood AM, Lewington S, Sattar N, et al. Major lipids, apolipoproteins, and risk of vascular disease. JAMA. 2009;302(18):1993-2000.

27. Sarwar N, Danesh J, Eiriksdottir G, Sigurdsson G, Wareham N, Bingham S, Boekholdt SM, Khaw KT, Gudnason V. Triglycerides and the risk of coronary heart disease: 10,158 incident cases among 262,525 participants in 29 Western prospective studies. Circulation. 2007;115(4):450-8.

28. Emerging Risk Factors C, Erqou S, Kaptoge S, Perry PL, Di Angelantonio E, Thompson A, White IR, Marcovina SM, Collins R, Thompson SG, et al. 
Lipoprotein(a) concentration and the risk of coronary heart disease, stroke, and nonvascular mortality. JAMA. 2009;302(4):412-23.

29. van der Harst $P$, Verweij $N$. Identification of 64 novel genetic loci provides an expanded view on the genetic architecture of coronary artery disease. Circ Res. 2018;122(3):433-43.

30. Toth PP. Cardiology patient page. The "good cholesterol": high-density lipoprotein. Circulation. 2005;111(5):e89-91.

31. Downs JR, Clearfield M, Weis S, Whitney E, Shapiro DR, Beere PA, Langendorfer A, Stein EA, Kruyer W, Gotto AM Jr. Primary prevention of acute coronary events with lovastatin in men and women with average cholesterol levels: results of AFCAPS/TeXCAPS. Air force/Texas coronary atherosclerosis prevention study. JAMA. 1998;279(20):1615-22.

32. Sever PS, Dahlof B, Poulter NR, Wedel H, Beevers G, Caulfield M, Collins R, Kjeldsen SE, Kristinsson A, Mclnnes GT, et al. Prevention of coronary and stroke events with atorvastatin in hypertensive patients who have average or lower-than-average cholesterol concentrations, in the AngloScandinavian cardiac outcomes trial--lipid lowering arm (ASCOT-LLA): a multicentre randomised controlled trial. Lancet. 2003;361(9364):1149-58,

33. Shepherd J, Cobbe SM, Ford I, Isles CG, Lorimer AR, MacFarlane PW, McKillop JH, Packard CJ. Prevention of coronary heart disease with pravastatin in men with hypercholesterolemia. West of Scotland coronary prevention study group. N Engl J Med. 1995;333(20):1301-7.

34. Liu HH, Guo YL, Wu NQ, Zhu CG, Gao Y, Qing P, Li S, Zhao X, Zhang Y, Sun $D$, et al. High-density lipoprotein cholesterol levels are associated with coronary severity but not with outcomes in new-onset patients with stable coronary artery disease. Atherosclerosis. 2017;263:104-11.

35. Musunuru K, Kathiresan S. Surprises from genetic analyses of lipid risk factors for atherosclerosis. Circ Res. 2016;118(4):579-85.

36. Cohen JC, Boerwinkle E, Mosley TH Jr, Hobbs HH. Sequence variations in PCSK9, low LDL, and protection against coronary heart disease. N Engl J Med. 2006;354(12):1264-72

37. Zhao Y, Chen J, Freudenberg JM, Meng Q, Rajpal DK, Yang X. Networkbased identification and prioritization of key regulators of coronary artery disease loci. Arterioscler Thromb Vasc Biol. 2016;36(5):928-41.

38. Wang IM, Zhang B, Yang X, Zhu J, Stepaniants S, Zhang C, Meng Q, Peters M, $\mathrm{He} \mathrm{Y,} \mathrm{Ni} \mathrm{C,} \mathrm{et} \mathrm{al.} \mathrm{Systems} \mathrm{analysis} \mathrm{of} \mathrm{eleven} \mathrm{rodent} \mathrm{disease} \mathrm{models} \mathrm{reveals} \mathrm{an}$ inflammatome signature and key drivers. Mol Syst Biol. 2012;8:594.

\section{Publisher's Note}

Springer Nature remains neutral with regard to jurisdictional claims in published maps and institutional affiliations.

Ready to submit your research? Choose BMC and benefit from:

- fast, convenient online submission

- thorough peer review by experienced researchers in your field

- rapid publication on acceptance

- support for research data, including large and complex data types

- gold Open Access which fosters wider collaboration and increased citations

- maximum visibility for your research: over $100 \mathrm{M}$ website views per year

At $\mathrm{BMC}$, research is always in progress.

Learn more biomedcentral.com/submissions 